\title{
Social interactions among epithelial cells during tracheal branching morphogenesis
}

\author{
Amin S. Ghabrial ${ }^{1} \&$ Mark A. Krasnow ${ }^{1}$
}

Many organs are composed of tubular networks that arise by branching morphogenesis in which cells bud from an epithelium and organize into a tube ${ }^{1-3}$. Fibroblast growth factors (FGFs) and other signalling molecules have been shown to guide branch budding and outgrowth ${ }^{4-7}$, but it is not known how epithelial cells coordinate their movements and morphogenesis. Here we use genetic mosaic analysis in Drosophila melanogaster to show that there are two functionally distinct classes of cells in budding tracheal branches: cells at the tip that respond directly to Branchless FGF and lead branch outgrowth, and trailing cells that receive a secondary signal to follow the lead cells and form a tube. These roles are not pre-specified; rather, there is competition between cells such that those with the highest FGF receptor activity take the lead positions, whereas those with less FGF receptor activity assume subsidiary positions and form the branch stalk. Competition appears to involve Notch-mediated lateral inhibition that prevents extra cells from assuming the lead. There may also be cooperation between budding cells, because in a mosaic epithelium, cells that cannot respond to the chemoattractant, or respond only poorly, allow other cells in the epithelium to move ahead of them.

The Drosophila tracheal system develops from epithelial sacs of about 80 cells from which primary, secondary and terminal branches sprout without cell division or cell death ${ }^{8,9}$. Primary branch sprouting is induced by Branchless (Bnl) FGF, a chemoattractant secreted by clusters of cells surrounding each $\operatorname{sac}^{4}$ (Fig. 1a), which activates Breathless (Btl) FGF receptor (FGFR), a receptor tyrosine kinase expressed on tracheal cells ${ }^{10}$. Primary branches contain 3-20 cells that organize into a tube as they migrate out from the sac (Fig. 1b). $\mathrm{Bnl}$ also induces the expression of secondary branching genes, such as the transcription factor pointed $(p n t)^{11}$, and specifies terminal cells at the ends of outgrowing branches ${ }^{4,8}$. Terminal cells ramify in the larva in response to Bnl to form fine terminal branches ${ }^{12}$. Other cells at the ends of primary branches become fusion cells that connect with neighbouring branches to form a continuous tracheal network. Terminal and fusion cell fate decisions are also influenced by the Notch, Dpp and Wingless signalling pathways ${ }^{13-17}$. Dorsal branches, the primary branches that we focus on here, typically consist of five or six cells: two cells near the branch tip, one (DB1) that becomes a terminal cell and another (DB2) that becomes a fusion cell, and three or four cells (DB3-DB6) that form the branch stalk (Fig. 1a, b).

In a genetic mosaic screen (A.S.G., B. P. Levi and M.A.K., unpublished observations), six mutants (724, 788, 1118, 1187, 1476 and 1684) were identified with a subtle phenotype: mosaic branches $(+/+,+/-,-/-$ cells) were grossly normal, yet homozygous mutant clones $(-/-$ cells $)$ rarely if ever included terminal cells (Fig. 1c-e, and Supplementary Tables S1-S4; data not shown for other primary branches). These were neither general nor terminalcell-specific lethal mutations because homozygous mutant cells were readily recovered in all other tracheal positions, and there was no decrease in the overall number of cells in mosaic dorsal branches
$(5.3 \pm 1.1$ (mean \pm s.d.) versus $4.9 \pm 1.1$ in contralateral control branches; $n=22$ pairs of branches) or the number of terminal cells (98\% of both wild-type $(n=127)$ and 724 or 788 mosaic dorsal branches $(n=291)$ had a terminal cell). It was difficult to imagine how mutations could block clone generation in specific cells. It seemed more likely that the mutations caused cells otherwise destined to become terminal cells to switch fates with other tracheal cells.

The six mutations compose a single lethal complementation group that mapped to the left arm of chromosome 3 and failed to complement breathless ${ }^{L G 18}$. DNA sequencing identified a single nucleotide change in each mutant resulting in a nonsense or missense mutation in $b t l$ (Fig. 2a). Five mutations (724, 788, 1118, 1476 and 1684) appear to be null $b t l$ mutations, whereas the sixth mutation (1187) causes partial loss of function (Fig. $2 \mathrm{~b}$ and data not shown). Thus, the 'no mutant terminal cells' gene is btl.

We quantified the distribution of cells homozygous for $b t l$ null mutations (724 and 788), or homozygous for a wild-type $b t l$ allele as a control, in mosaic dorsal branches (Supplementary Tables S1-S4). Control clones were evenly distributed throughout the branch at the expected frequencies; for example, the ratio of stalk-cell to terminalcell clones was about $3: 1$. By contrast, $b t l^{-1-}$ cells showed a nearly complete bias against the DB1 position: the ratio of stalk-cell to terminal-cell clones was 51:1. The three exceptional mutant terminal cells may be cases in which the clone was induced after $b t l$ began to be expressed, allowing wild-type $b t l$ gene products to perdure in mutant cells. We recovered hundreds of mosaic branches with one or more $b t l^{-/-}$cells present in positions DB2-DB6 without affecting cell or branch morphology. Indeed, branches composed largely or exclusively of $b t l^{-1-}$ cells, except for a wild-type terminal cell, were morphologically indistinguishable from wild-type branches (Fig. 2c, and Supplementary Tables S3 and S4). Thus, although all tracheal cells normally express $b t l$, and the receptor is activated by Bnl in most or all cells of budding branches ${ }^{8,18}$, the receptor appears to be required in just a single leading cell (DB1). All other cells can migrate normally and form tubes in the absence of $b t l$. We conclude that there are two functionally distinct classes of cells in budding primary branches: lead cells, which require Btl FGFR and directly respond to Bnl FGF, and trailing cells, which do not require Btl but follow the lead cell and form the stalk.

What does it take to become the leader? The lead cell (DB1) is specified to become a terminal cell by Bnl-Btl signalling ${ }^{4,8}$. If terminal cell specification is required, then null mutations in the downstream gene pnt, which abolish this function ${ }^{8}$, should have the same effect as $b t l$ mutations. Cell clones homozygous for $p n t^{\Delta 88}$ or two new pnt alleles isolated in our screen (198 and 1318) failed to develop as terminal cells, as expected. However, unlike $b t l$ mosaic branches, pnt mosaic branches often lacked a terminal cell (26\% of mosaic branches; $n=97)$. When a terminal cell was missing, there was usually (about $80 \%$ of the time) a $p n t^{-1-}$ cell in the stalk position nearest the tip (Fig. 2d, and Supplementary Table S5), presumably 
a

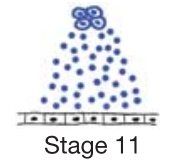

$(6 \mathrm{~h})$

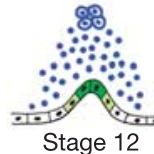

$(8 \mathrm{~h})$

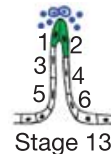

(10 h)

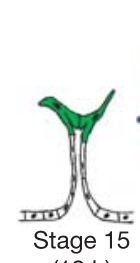

(12 h)

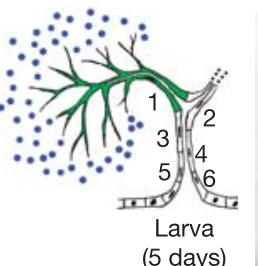

(5 days)
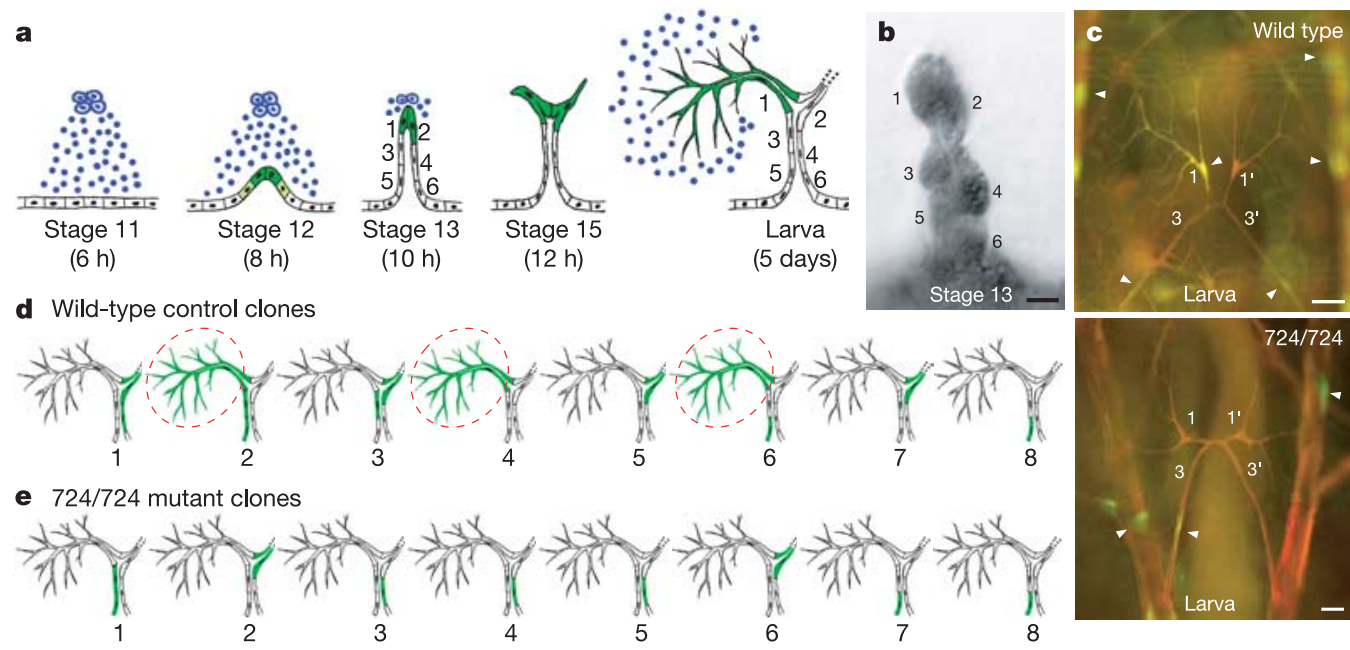

Figure 1 | The 'no mutant terminal cells' phenotype. a, Diagram of dorsal branch (DB) budding from tracheal epithelium (black; DB cells numbered 1-6) at the developmental stages and times indicated. Nearby cells (blue) secrete Branchless FGF (blue dots), which activates Breathless (Btl) FGFR on tracheal cells, inducing migration and tube formation. Bnl also induces secondary branching genes (for example pointed) in cells (green) that form unicellular secondary branches (stage 15). Subsequently, DB1 (terminal cell) forms terminal branches in response to Bnl expressed by hypoxic larval cells. DB2 (fusion cell) forms a branch that fuses (dotted lines) to a contralateral DB (not shown). DB3-6 cells form DB stalk. b, Micrograph of budding DB (stage 13). Nuclei are black; cytoplasm is grey. Cells here are arranged side by side, but subsequently the stalk cells intercalate. Reprinted

with permission (ref. 8). Scale bar, $2.5 \mu \mathrm{m}$. c, Fluorescence micrograp
GFP-marked clones in pairs of larval DBs (dorsal view, anterior up). Tracheal cells are labelled with DsRED (red), and homozygous clones are labelled with GFP (green). Arrowheads indicate select clones in DBs and dorsal trunks (large branches flanking DBs). Top panel: control wild-type clones. Bottom panel: homozygous 724 mutant clones. DB1 $\left(1,1^{\prime}\right)$ and DB3 $\left(3,3^{\prime}\right)$ cells in left and right DBs are indicated. Scale bars, $50 \mu \mathrm{m}$.

d, Distribution of control clones (green) in eight representative DBs. Three contain DB1/terminal cell clones (circled). For simplicity, only DBs with five cells (DB1-DB5) are shown. e, Distribution of homozygous 724 mutant clones in eight representative DBs. Clones had no morphological defects but were excluded from DB1 positions. the DB1 cell that failed to differentiate into a terminal cell. This suggests that $p n t^{-1-}$ cells are able to assume the lead position but fail to differentiate as terminal cells, and that the bias against btl mutant terminal cells is due to the earlier, pnt-independent, function of Btl in primary branch budding and outgrowth. If cells lacking Btl cannot migrate in response to Bnl during budding ${ }^{8,10,19}$, they should not be able to move to the lead position necessary to be selected as a terminal cell. Consistent with this, genetic mosaic analysis of stumps (dof/heartbroken), which encodes a Btl adaptor required for cell migration ${ }^{20,21}$, showed a dearth of terminal cell clones similar to $b t l$ (data not shown).

Two results demonstrate that the ability to sense Bnl and migrate in response to it is not enough to become the leader: cells compete for the lead position. The first involves $b t l^{B N}$ (E796K mutation in the kinase domain), a weak $b t l$ allele isolated in a separate screen. Unlike $b t l^{-1-}$ animals, which die in first larval instar and lack virtually all branches ${ }^{10}$ (Fig. 2b), $b t l^{B N}$ homozygotes survived until L3 larval stage or beyond and had a normally patterned tracheal system with a full complement of terminal cells. The only defects detected were a reduced number and altered morphology of terminal branches, presumably due to the dosage-sensitive function of $b t l$ in terminal branch outgrowth ${ }^{12}$ (Fig. 3a-c). The late and subtle phenotype demonstrates that $\mathrm{Bt}{ }^{\mathrm{BN}}$ protein retains sufficient activity for early migration and terminal cell specification events. However, we found that in genetic mosaic animals, in which $b t l^{B N / B N}$ cells must compete with $b t l^{B N /+}$ and $b t l^{+/+}$tracheal cells, $b t l^{B N / B N}$ cells rarely acquired the lead position (DB1) and developed as terminal cells. Indeed, homozygosity for $b t l^{B N}$ conferred nearly as complete a bias against becoming a terminal cell as total loss of btl (Fig. 3d-f, and Supplementary Tables S1 and S6). Thus, Btl activity above the threshold necessary for migration and terminal cell specification is not sufficient to acquire the lead position and become a terminal cell: a cell must have more Btl activity than other cells in the branch.

Similar conclusions derive from a second experiment in which we analysed marked wild-type $\left(b t l^{+l+}\right)$ cells in heterozygous $\left(b t l^{+l-}\right)$ animals. Whereas $b t l^{+/+}$clones in control $\left(b t l^{+/+}\right)$animals were distributed evenly throughout the branch (Fig. $3 \mathrm{~d}$ ), $b t l^{+/+}$clones in $b t l^{788 /+}$ heterozygotes preferentially localized to the tip (Fig. 3g, and Supplementary Tables S1, S2 and S7). Cells that did not occupy the lead (DB1) position took positions close to the tip (Fig. 3g, and Supplementary Table S7). Similar results were obtained for $b t l^{+/+}$ clones in animals heterozygous for $b t l^{1187}$, a partial-loss-of-function allele (Fig. 3h, and Supplementary Tables S1 and S8). Clones mutant for sprouty, an FGF feedback inhibitor ${ }^{22,23}$, also preferentially populated the tip (Supplementary Table S9). Together, the data show that there is competition for the lead position: cells with highest $b t l$ activity assume positions at or near the tip of the branch, whereas those with less or no activity segregate towards its base.

Because small differences in btl dosage or activity affect a cell's ability to compete for the lead, we investigated whether lateral inhibitory mechanisms that amplify small differences in signalling might be operative. Data suggest that the Notch pathway, a lateral signalling pathway implicated in cell specification events including cell fate determination at tracheal branch tips, also affects cell arrangement $t^{15-17} . N^{t s}$ embryos shifted to the restrictive temperature during budding formed branches in which most DB cells behaved like lead cells, resulting in large clusters of cells congregated at the lead position ${ }^{17}$, whereas expression of constitutively active $\mathrm{N}^{\mathrm{ACT}}$ throughout the tracheal epithelium had the opposite effect, arresting outgrowth and stalling cells near the base of the branch (Fig. 4a-c). We propose that Notch-mediated lateral inhibition among tracheal cells prevents extra cells from assuming the lead position.

Our results provide evidence for social stratification and dynamic social interactions between epithelial cells during branching morphogenesis (Fig. 4d). First, the results show that budding cells are functionally specialized. A cell at the branch tip requires $b t l$ and leads outgrowth towards the Bnl signalling centre. Trailing cells do not require $b t l$ but nevertheless follow the lead cell towards the Bnl source. Because tracheal cells do not migrate or form tubes in $b t l^{-1-}$ animals, trailing cells must receive a secondary signal generated by the lead cell that induces them to migrate and also activates their tubulogenesis programme. This could be a secreted molecule or physical stimulus such as pulling or stretching the trailing cells. 
a
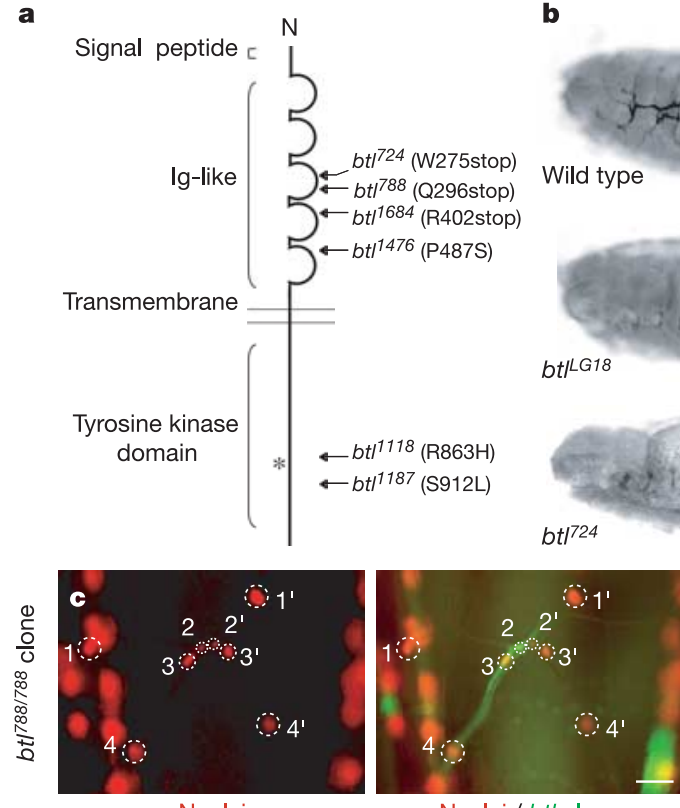

Nuclei / btl clone

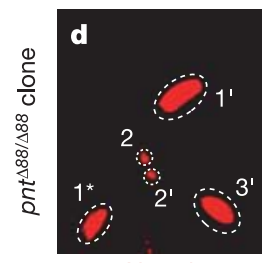

Nuclei
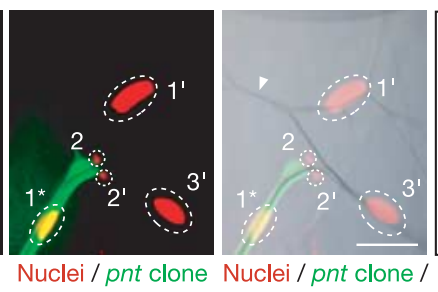

$\mathrm{DIC}$

Figure 2 'No mutant terminal cells' mutations are loss-of-function mutations in btl. a, Changes in btl coding sequence in 'no mutant terminal cells' mutants. Asterisk, kinase active site. b, Wild-type, homozygous $b t l^{L G 18}$ and homozygous $b t l^{724}$ embryos (10-13 h old) immunostained with tracheal-specific monoclonal antibody 2A12. Lateral views, anterior left, dorsal up. Note the absence of branches in both mutants. Scale bar, $50 \mu \mathrm{m}$. c, Fluorescence micrographs of mosaic DB with large $b t l^{788 / 788}$ clone (dorsal view). Tracheal nuclei are labelled with DsRed2nls (red; left and middle panels), and DB cell nuclei are circled (DB1-DB4, mosaic DB; DB1'-DB4', contralateral control DB). (DB1 staining is partly obscured by underlying dorsal trunk staining.) Homozygous $b t l^{788 / 788}$ cells are labelled with GFP (green; middle panel) and comprise the entire mosaic DB except DB1 (see diagram, right panel), yet DB has a normal morphology, like the contralateral control DB. Scale bar, $50 \mu \mathrm{m}$. d, Mosaic DB containing a null pointed $^{\Delta 88}$ clone (dorsal view). First panel: DsRed2nls-labelled nuclei (red) are circled $\left(\mathrm{DB}^{\star}{ }^{\star}\right.$ and $\mathrm{DB} 2$, mosaic $\mathrm{DB}$; $\mathrm{DB} 1^{\prime}-\mathrm{DB} 3^{\prime}$, contralateral control $\mathrm{DB})$. Second panel: $p n t^{\Delta 88 / \Delta 88}$ clone (green, $\mathrm{DB} 1^{\star}$ ) did not develop as a terminal cell or assume its normal position; instead it formed the distal cell of the stalk. Third panel: red and green channels superimposed on a differential interference contrast image, showing air-filled lumens of DBs. DB1' extends terminal branches (arrowhead) into region normally supplied by missing terminal cell. Fourth panel: diagram of mosaic DB, with pnt ${ }^{\Delta 88 / \Delta 88}$ clone shaded green. Scale bar, $50 \mu \mathrm{m}$.

Second, these roles are not pre-specified. Rather, there is competition between cells such that those with high Btl FGFR activity become lead cells whereas those with less or no btl FGFR activity become trailing cells and form the branch stalk. Competition appears to involve Notch-mediated lateral inhibitory signalling between tracheal cells, and it may also be influenced by positive feedback mechanisms such as increased activation and expression of Btl as cells approach the Bnl source ${ }^{24}$. Third, there may be cooperation between cells, because in a genetically mosaic epithelium, tracheal cells with less Btl activity allow those with more activity to move ahead of them.

There may be similar social interactions between budding cells in other branching organs. Studies of other branching processes have identified genes selectively expressed in tip cells of budding branches,

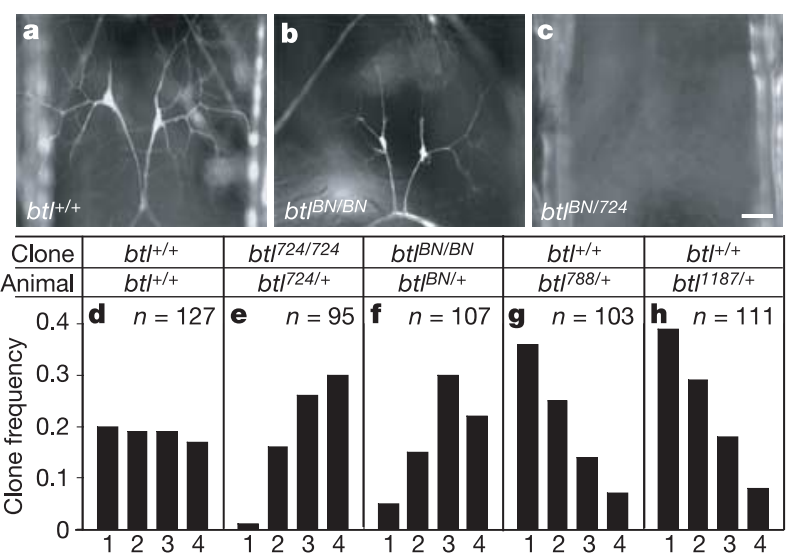

Position of cell in dorsal branch

Figure 3 Effect of btl dosage on DB cell position. a-c, Fluorescence micrographs of DB pair (dorsal view) in control $b t l^{+}$larva (a), larva homozygous for the weak hypomorphic allele $b t l^{B N}(\mathbf{b})$ and larva transheterozygous for $b t l^{B N}$ and null $b t l^{724}$ allele (c). Tracheal cells were labelled by expression of a UAS-GFP transgene driven by $b t l-G a l 4$ $(b t l-G a l 4>\mathrm{GFP})$. The $b t l^{B N / B N}$ larva has normal DB primary and secondary branches, and a normal number of terminal cells, although terminal branching is reduced. $b t l^{B N / 724}$ lacks DBs. Scale bar, $50 \mu \mathrm{m}$. $\mathbf{d}-\mathbf{h}$, Relative frequency of GFP-marked clones at different DB positions. Genotypes of clones and animals in which clones were induced are indicated. $n$, number of DBs analysed. Because the average number of cells per clone varied in different experiments $(\mathbf{d}, 1.67 ; \mathbf{e}, 1.33 ; \mathbf{f}, 1.36 ; \mathbf{g}, 1.52 ; \mathbf{h}, 1.55)$, clone frequencies shown are relative values normalized to one GFP-marked cell per branch. $\mathbf{d}$, Control clones were evenly distributed between positions DB1-DB4. e, Clones with no $b t l$ activity $\left(b t l^{724 / 724}\right)$ induced in $b t l^{724 /+}$ animals showed a graded distribution, rarely in DB1 position and increasing in frequency towards the branch base. $f$, Clones with mildly reduced $b t l$ activity $\left(b t l^{B N / B N}\right)$ induced in $b t l^{B N /+}$ animals showed a similar distribution, even though $b t t^{B N / B N}$ cells populate all positions in $b t l^{B N / B N}$ animals (b). $\mathbf{g}, \mathbf{h}, b t l^{+/+}$clones induced in animals heterozygous for null $b t l^{788}$ allele $(\mathbf{g})$ or moderate $b t l^{1187}$ allele (h) showed the opposite distribution, preferentially in DB1 positions and decreasing in frequency towards the branch base.

and in some cases these cells display morphological specializations indicating that they might actively lead outgrowth ${ }^{25}$. However, because most budding branches contain hundreds or thousands of cells, it is difficult to track and manipulate individual cells to investigate social behaviours like those described here. Recent analyses of chimaeric $\operatorname{Ret}^{+} / \operatorname{Ret}^{-}$mouse renal ureteric buds in culture $^{26}$ and $b t l$ mosaic air sacs ${ }^{27}$ reveal that cells lacking these receptor tyrosine kinases are excluded from branch tips, indicating that RTK-dependent interactions similar to those described here might be operative in more complex branching events.

\section{METHODS}

Fly stocks. $b t l^{L G 18}, s p r y^{\Delta 5}, p n t^{\Delta 88}$ and $N^{l 1 N-t s 1}\left(\right.$ abbreviated $\left.N^{t s}\right)$ are described in Flybase (http://flybase.bio.indiana.edu/). New btl and pnt alleles were generated by ethane methyl sulphonate mutagenesis of a third chromosome containing FLP recombinase sites FRT2A and FRT82B (Flybase) and isolated in screens described elsewhere (A.S.G., B. P. Levi and M.A.K., unpublished observations). The Gal4UAS system ${ }^{28}$ with $b t$-GAL4 driver (Flybase) was used to express proteins throughout the tracheal system. UAS responders were as follows: UAS-DsRED (A.S.G., B. P. Levi and M.A.K., unpublished observations), which expresses a cytoplasmic red fluorescent protein; UAS-DsRED2nls (M. Galko, G. Fish and M.A.K, unpublished observations), which expresses a nuclear DsRed2 protein; UAS-GFP (Flybase); UASi-GFPhp (A.S.G., B. P. Levi and M.A.K., unpublished observations), which expresses a double-stranded hairpin RNA that inhibits GFP expression by RNA-mediated interference; and UAS-N ${ }^{\text {ACT }}$ (Flybase).

Clone generation and labelling. Heterozygous embryos ( $1-3 \mathrm{~h}$ old $)$ carrying the hsFLP ${ }^{1.22}$ recombinase transgene (Flybase) were reared at $25^{\circ} \mathrm{C}$ and then placed at $38^{\circ} \mathrm{C}$ for $45-60 \mathrm{~min}$ to induce mitotic recombination at FRT sites centromereproximal to the mutation, generating clones of homozygous mutant cells ${ }^{29}$. Tracheal cells were marked with a btl-GAL4 transgene to drive expression of UAS-DsRED (or UAS-DsRED2nls) and UAS-GFP transgenes. Expression of the latter was blocked by a UASi-GFPhp transgene present in trans to the mutation 

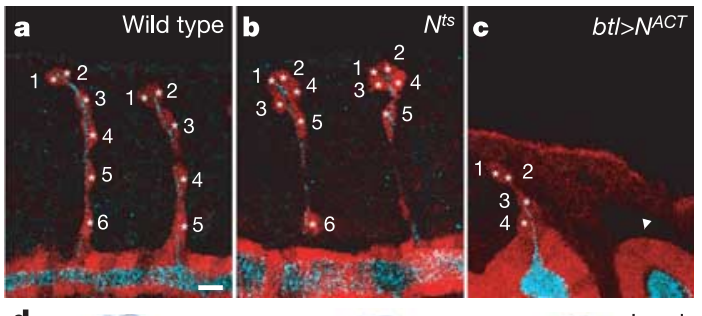

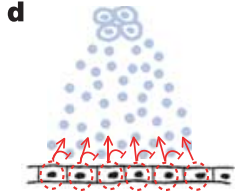

Competition

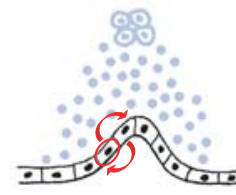

Cooperation

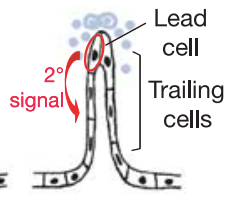

Communication
Figure 4 | Effects of Notch $(N)$ activity on cell position. a-c, Fluorescent micrographs of two DBs (lateral view) in stage 15 wild-type embryo (a), $N^{t s}$ embryo shifted to non-permissive temperature for $6 \mathrm{~h}$ during branch budding (b) and $b t \mathrm{lGal} 4>\mathrm{UAS}-N^{A C T}$ embryo that expressed activated $\mathrm{N}$ throughout the tracheal system (c). All embryos carried $b t l$ Gal4 and UASGFP transgenes and were double-stained for GFP (red; tracheal cell marker) and Vermiform (cyan; luminal marker). a, Cells in wild-type DBs are evenly distributed (nuclei are numbered and indicated by asterisks). b, $N$ inactivation caused the migration of extra cells to the DB tip. c, Constitutive $N$ activity inhibited outgrowth, particularly in posterior metameres in which some DBs completely failed to bud (arrowhead). Scale bar, $5 \mu \mathrm{m}$. d, Social interactions between tracheal cells during budding. The three panels show budding tracheal cells expressing the Btl FGFR moving towards a Bnl FGF signalling centre, as in Fig. 1a. The first panel illustrates cell competition: cells move towards the lead position and inhibit their neighbours from doing the same. The second panel illustrates cell cooperation: a cell with less Btl activity allows one with more to move ahead of it. The third panel illustrates cell communication: the lead cell sends a secondary $\left(2^{\circ}\right)$ signal to the trailing cells, inducing them to follow the lead cell and activating a tubulogenesis programme. Cells also communicate via Notch-mediated signalling as they compete for the lead position (inhibition arrow in first panel).

of interest. Homozygous mutant tracheal cells lack the UASi-GFPhp transgene and so express GFP as well as DsRED. After clone induction, embryos were returned to $25^{\circ} \mathrm{C}$, and after four or five days the third instar larvae were heatkilled $\left(70^{\circ} \mathrm{C}\right.$ for $\left.3-5 \mathrm{~s}\right)$, mounted in $50 \%$ glycerol and observed by fluorescence microscopy to score clone distribution. See Supplementary Methods for complete descriptions of genotypes and clone analysis.

Tracheal cell counts. Mosaic dorsal branches containing at least one homozygous, GFP-expressing cell were scored for presence of terminal (DB1) cells by morphological criteria. Cell numbers in mosaic branches and in contralateral control branches were determined by counting DsRED2nls-labelled nuclei. Notch experiments. $N^{t s} ; b t l-G a l 4$, UAS-GFP/+ embryos were collected at $18^{\circ} \mathrm{C}$ for $3 \mathrm{~h}$, aged for $10 \mathrm{~h}$, shifted to the non-permissive temperature $\left(30.5^{\circ} \mathrm{C}\right)$ for $6 \mathrm{~h}$, then fixed, immunostained and analysed by confocal microscopy. For expression of $\mathrm{N}^{\mathrm{ACT}}$ throughout the tracheal system, $b t l-G a 4$, UAS-GFP/+; $b t l-G a 4$, UASCD8GFP/UAS- $\mathrm{N}^{\mathrm{ACT}}$ embryos were raised at $30.5^{\circ} \mathrm{C}$.

Immunostaining and microscopy. Embryo fixation and tracheal staining with monoclonal antibody $2 \mathrm{~A} 12$ was performed as described ${ }^{8}$. For Fig. 4, embryos were fixed and double-stained with goat anti-GFP (1:200 dilution; Abcam) and anti-Verm ${ }^{30}$ (1:600 dilution), and revealed by indirect immunofluorescence with fluorescein isothiocyanate-conjugated and CY3-conjugated secondary antibodies. Stained embryos and labelled tracheal clones were analysed and photographed with compound or confocal fluorescence microscopes. Where necessary, a montage of optical sections was assembled (Figs 1c and 4a-c).

\section{Received 30 October 2005; accepted 19 April 2006}

1. Hogan, B. L. \& Kolodziej, P. A. Organogenesis: molecular mechanisms of tubulogenesis. Nature Rev. Genet. 3, 513-523 (2002).

2. Metzger, R. J. \& Krasnow, M. A. Genetic control of branching morphogenesis Science 284, 1635-1639 (1999).

3. Affolter, M. et al. Tube or not tube: remodeling epithelial tissues by branching morphogenesis. Dev. Cell 4, 11-18 (2003).

4. Sutherland, D., Samakovlis, C. \& Krasnow, M. A. branchless encodes a Drosophila FGF homolog that controls tracheal cell migration and the pattern of branching. Cell 87, 1091-1101 (1996).

5. Park, W. Y., Miranda, B., Lebeche, D., Hashimoto, G. \& Cardoso, W. V. FGF-10 is a chemotactic factor for distal epithelial buds during lung development. Dev. Biol. 201, 125-134 (1998).

6. Weaver, M., Dunn, N. R. \& Hogan, B. L. Bmp4 and Fgf10 play opposing roles during lung bud morphogenesis. Development 127, 2695-2704 (2000).

7. Pepicelli, C. V., Kispert, A., Rowitch, D. H. \& McMahon, A. P. GDNF induces branching and increased cell proliferation in the ureter of the mouse. Dev. Biol. 192, 193-198 (1997).

8. Samakovlis, C. et al. Development of the Drosophila tracheal system occurs by a series of morphologically distinct but genetically coupled branching events. Development 122, 1395-1407 (1996)

9. Ghabrial, A., Luschnig, S., Metzstein, M. M. \& Krasnow, M. A. Branching morphogenesis of the Drosophila tracheal system. Annu. Rev. Cell Dev. Biol. 19 623-647 (2003)

10. Klambt, C., Glazer, L. \& Shilo, B. Z. breathless, a Drosophila FGF receptor homolog, is essential for migration of tracheal and specific midline glial cells. Genes Dev. 6, 1668-1678 (1992).

11. Klambt, C. The Drosophila gene pointed encodes two ETS-like proteins which are involved in the development of the midline glial cells. Development 117 163-176 (1993).

12. Jarecki, J., Johnson, E. \& Krasnow, M. A. Oxygen regulation of airway branching in Drosophila is mediated by branchless FGF. Cell 99, 211-220 (1999).

13. Llimargas, M. Wingless and its signalling pathway have common and separable functions during tracheal development. Development 127, 4407-4417 (2000).

14. Chihara, T. \& Hayashi, S. Control of tracheal tubulogenesis by Wingless signaling. Development 127, 4433-4442 (2000).

15. Llimargas, M. The Notch pathway helps to pattern the tips of the Drosophila tracheal branches by selecting cell fates. Development 126, 2355-2364 (1999).

16. Ikeya, T. \& Hayashi, S. Interplay of Notch and FGF signaling restricts cell fate and MAPK activation in the Drosophila trachea. Development 126, 4455-4463 (1999).

17. Steneberg, P., Hemphala, J. \& Samakovlis, C. Dpp and Notch specify the fusion cell fate in the dorsal branches of the Drosophila trachea. Mech. Dev. 87, 153-163 (1999).

18. Gabay, L., Seger, R. \& Shilo, B. Z. MAP kinase in situ activation atlas during Drosophila embryogenesis. Development 124, 3535-3541 (1997).

19. Ribeiro, C., Ebner, A.\& Affolter, M. In vivo imaging reveals different cellular functions for FGF and Dpp signaling in tracheal branching morphogenesis. Dev. Cell 2, 677-683 (2002).

20. Vincent, S., Wilson, R., Coelho, C., Affolter, M. \& Leptin, M. The Drosophila protein Dof is specifically required for FGF signaling. Mol. Cell 2, 515-525 (1998).

21. Imam, F., Sutherland, D., Huang, W. \& Krasnow, M. A. stumps, a Drosophila gene required for fibroblast growth factor (FGF)-directed migrations of tracheal and mesodermal cells. Genetics 152, 307-318 (1999).

22. Hacohen, N., Kramer, S., Sutherland, D., Hiromi, Y. \& Krasnow, M. A. sprouty encodes a novel antagonist of FGF signaling that patterns apical branching of the Drosophila airways. Cell 92, 253-263 (1998).

23. Casci, T., Vinos, J. \& Freeman, M. Sprouty, an intracellular inhibitor of Ras signaling. Cell 96, 655-665 (1999).

24. Ohshiro, T., Emori, Y. \& Saigo, K. Ligand-dependent activation of breathless FGF receptor gene in Drosophila developing trachea. Mech. Dev. 114, 3-11 (2002)

25. Gerhardt, H. G., Alitalo, K., Shima, D., Betsholtz, C. et al. VEGF guides angiogenic sproutting utilizing endothelial tip cell filopodia. J. Cell Biol. 161, 1163-1177 (2003).

26. Shakya, R., Watanabe, T. \& Costantini, F. The role of GDNF/Ret signaling in ureteric bud cell fate and branching morphogenesis. Dev. Cell 8, 65-74 (2005).

27. Cabernard, C. \& Affolter, M. Distinct roles for two receptor tyrosine kinases in epithelial branching morphogenesis in Drosophila. Dev. Cell 9, 831-842 (2005).

28. Brand, A. H. \& Perrimon, N. Targeted gene expression as a means of altering cell fates and generating dominant phenotypes. Development 118, 401-415 (1993)

29. Golic, K. G. Site-specific recombination between homologous chromosomes in Drosophila. Science 252, 958-961 (1991).

30. Luschnig, S., Batz, T., Armbruster, K. \& Krasnow, M. A. serpentine and vermiform encode matrix proteins with chitin binding and deacetylation domains that limit tracheal tube length in Drosophila. Curr. Biol. 16, 186-194 (2006).

Supplementary Information is linked to the online version of the paper at www.nature.com/nature.

Acknowledgements We thank B. Levi for help with isolation of 'no mutant terminal cell' mutants and for discussion, and S. Artavanis, M. Galko, S. Luschnig and S. Toering for fly stocks and antisera. This work was supported by an NIH NRSA fellowship (A.S.G.) and a grant from the N.I.H. M.A.K. is an investigator of the Howard Hughes Medical Institute.

Author Contributions A.S.G. conceived, designed and performed experiments and analysed data. M.A.K. advised on the above. A.S.G. and M.A.K. wrote the paper.

Author Information Reprints and permissions information is available at npg.nature.com/reprintsandpermissions. The authors declare no competing financial interests. Correspondence and requests for materials should be addressed to M.A.K. (krasnow@cmgm.stanford.edu) 